\title{
E2FBP1/hDril1 modulates cell growth through downregulation of promyelocytic leukemia bodies
}

\author{
Y Fukuyo ${ }^{1,2}$, K Mogi $^{1}$, Y Tsunematsu ${ }^{2}$ and T Nakajima ${ }^{*, 1}$ \\ 1 Department of Molecular Cellular Oncology and Microbiology, Graduate \\ School, Tokyo Medical and Dental University, 1-5-45 Yushima, Bunkyo-ku, \\ Tokyo 113-8549, Japan \\ 2 Faculty of Applied Biological Science, Tokyo University of Science, 2641 \\ Yamazaki, Noda-shi, Chiba 278-8510, Japan \\ * Corresponding author: T Nakajima. Tel.: + 81358035458 \\ Fax: + 8145982 7141; E-mail: nakajima.mcom@tmd.ac.jp
}

Received 29.5.03; revised 02.10.03; accepted 06.1.04; published online 12.3.04 Edited by $\mathrm{H}$ Ichijo

\begin{abstract} depots.

Cell Death and Differentiation (2004) 11, 747-759.

doi:10.1038/sj.cdd.4401412

Published online 12 March 2004
\end{abstract}

Promyelocytic leukemia nuclear bodies (PML-NBs) comprise multiple regulatory factors and play crucial roles in the maintenance of cellular integrity, while unregulated activation of PML-NBs induces death and premature senescence. Hence, the function of PML-NBs must be directed properly; however, the mechanism that regulates PML-NBs remains unclear. In this paper, we show that PML-NBs are disintegrated by an AT-rich interaction domain family protein E2FBP1/hDril1 through specific desumoylation of promyelocytic leukemia protein (PML) in vivo and in vitro. RNA interference-mediated downregulation of E2FBP1/hDril1 results in hyperplasis of PML-NBs and consequent commitment to PML-dependent premature senescence. Thus, the function of E2FBP1/hDril1 is required for maintenance of survival potential of the cells. Our data suggest a novel mechanism to govern cellular integrity through the modulation of nuclear

Keywords: PML nuclear body; premature senescence; proteinprotein interaction; sumoylation

Abbreviations: IgH, immunoglobulin heavy chain; ND10, nuclear domain 10; pRb, retinoblastoma susceptible gene product; IP, immune complex; DOC, deoxycholic acid sodium salt; ORF, open reading frame; RNAi, RNA interference; PAl-1, plasminogen activator inhibitor-1; SA- $\beta$-gal, senescence-associated $\beta$-galactosidase

\section{Introduction}

E2FBP1 was cloned as an E2F-binding protein by Westwestern screening and characterized as a ubiquitously expressed human enhancer protein for E2F-mediated transactivation; ${ }^{1}$ however, the details of its function remain obscure. Database search revealed E2FBP1 to be identical to an AT-rich interaction domain (ARID) family DNA-binding protein, hDril1, which was isolated as a human orthologue of the mouse B-cell regulator of IgH transcription, Bright, and the Drosophila dead ringer protein, $\mathrm{Dri}^{2}{ }^{2}$ These close homologues of E2FBP1/hDril1 (hereafter, simplified to 'E2FBP1') share a highly homologous ARID with extended flanking regions (eARID). Although few functional overlap has been reported, eARID family proteins are appreciated for their essential roles in the regulation of species and/or cell type-specific responses. ${ }^{3}$ In addition to the eARID, E2FBP1 retains another conserved motif REKLES, that is accepted as an important motif for oligomerization, ${ }^{3}$ within a putative Myc-type helixloop-helix (HLH) domain. ${ }^{1}$ In this context, E2FBP1 was thought to be implicated in a cellular control system through these domains. To elucidate the function of E2FBP1, we sought the factors that interact with E2FBP1 using the yeast two-hybrid system and identified two factors that compose promyelocytic leukemia nuclear bodies (PML-NBs): speckled protein 100 (Sp100) and a conjugation enzyme for small ubiquitin-like modifier (SUMO)-1, UbcH9. ${ }^{4}$ Since Sp100 was reported to interact with Bright to suppress expression of the $\mathrm{IgH}$ gene, ${ }^{5,6}$ our findings suggested a functional interaction between E2FBP1 and Sp100. In contrast to Bright, whose expression is limited in lineage of rodent lymphoid cells, ${ }^{7}$ the expression of E2FBP1 is ubiquitous. Thus, interaction between E2FBP1 and either component of PML-NBs is likely to be involved in a general cellular function.

PML-NBs (also known as nuclear dots (NDs), nuclear domain 10 (ND10) or promyelocytic leukemia oncogenic domains (PODs)) are one of the distinct class of annular structures found in mammalian cell nucleus, named as nuclear bodies (NBs). Although their function has not been fully elucidated, it becomes clear that PML-NBs contribute to the multiple cellular responses to diverse stimuli including genotoxic stress, viral infections, oncogenic disorders and extracellular signals. ${ }^{8}$ Upon those cellular responses, PMLNBs change numbers, size and composition; hence its architecture should be dynamically regulated. The major components of PML-NBs are promyelocytic leukemia protein (PML) and Sp100, and the proapoptotic adaptor protein Daxx plays a crucial role in the assembly of PML-NBs by connecting sumoylated PML and Sp100. ${ }^{9-11}$ These provide a scaffold to harbor several prominent regulatory factors including tumor suppressors, transcriptional regulators, and enzymes for signal transduction, and thereby PML-NBs contribute to multiple mechanisms of cellular control. ${ }^{8,10,12-16}$ While the functional interactions of these components remain unclear, the architecture of PML-NBs seems to be particularly important since its abrogation caused by a chromosomal translocation $t(15 ; 17)$ results in acute promyelocytic leukemia. ${ }^{8,14,16}$ In contrast, forced expression of PML, as well as the expression of oncogenic Ras, induces apparent hyperplasis (i.e. increase in both size and number) of PML-NBs and subsequent commitment to premature senescence. ${ }^{17,18}$ Since both a dysfunction and unregulated hyperplasis of PML-NBs affect cellular integrity and proliferative potential, 
the mechanism regulating PML-NBs should be implicated in the cellular governance system.

In this paper, we show that E2FBP1 disintegrates PMLcomplex in vivo and in vitro, and RNAi-mediated downregulation of E2FBP1 commits primary human fibroblast to premature senescence via hyperplasis of PML-NBs. Our results strongly suggest that E2FBP1 modulates cell growth through the downregulation of PML-NBs.

\section{Results}

\section{E2FBP1 interacts with components of PML-NBs}

To identify factors that interact with E2FBP1, a low-background yeast two-hybrid selection method was applied using the host strain PJ69-4A. ${ }^{19}$ A total of $2.2 \times 10^{6}$ clones of normal human liver cDNA linked to the GAL4 activation domain were screened against E2FBP1 linked to the GAL4 DNA-binding

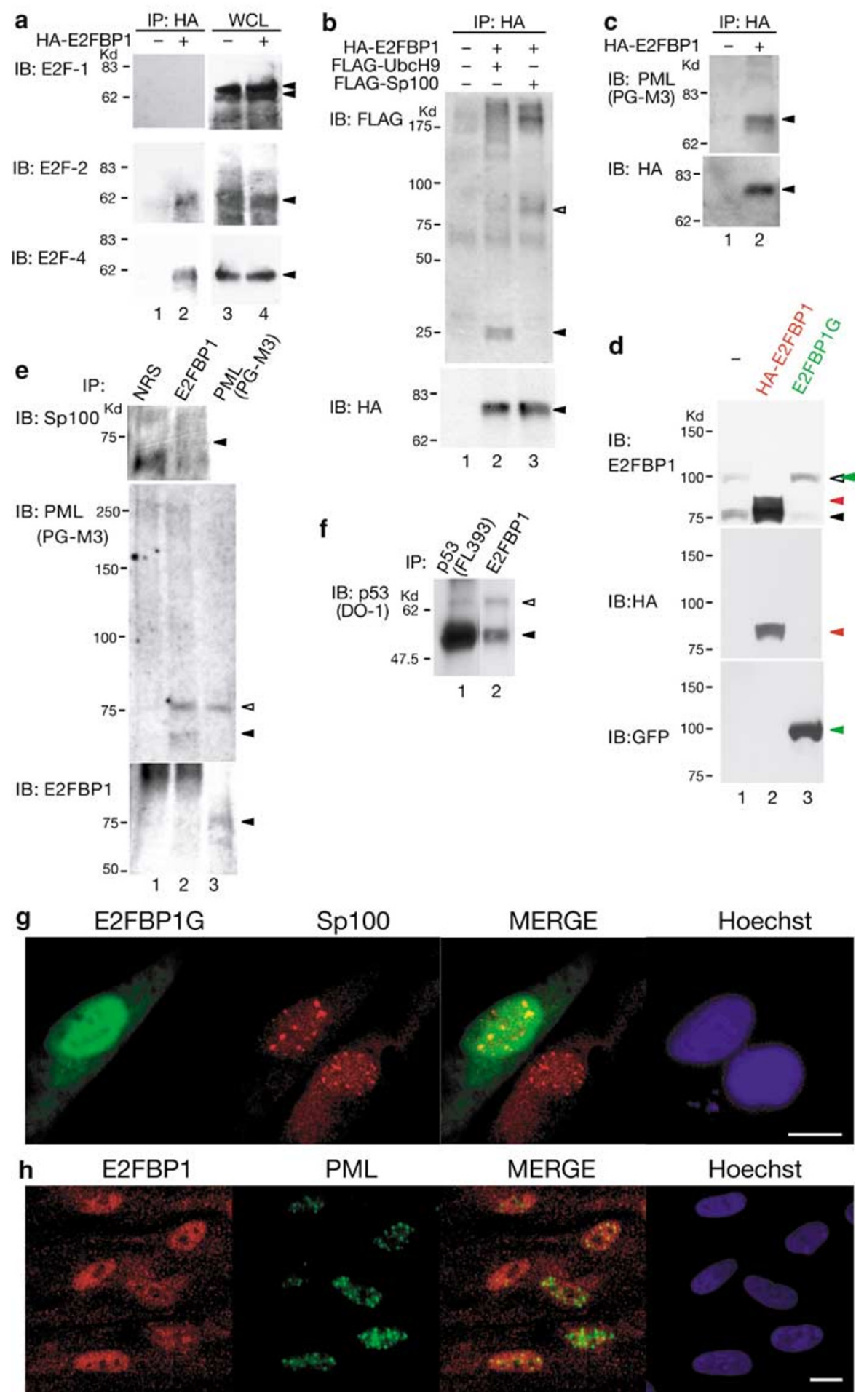


domain (E2FBP1-BD). Six independently raised clones showed significant interaction with E2FBP1-BD. Two clones encoded UbcH9, two clones encoded Sp100 and the remaining two clones encoded short peptides (data not shown). Although interaction between E2FBP1 and E2F-1 had been detected in the human embryonic carcinoma cell line $\mathrm{NEC} 14,{ }^{1}$ no E2F family members were found in the screening. The result implied that the interaction seen in yeast cells might not exactly reflect that in human cells, whereas a close homologue of E2FBP1, Bright, was reported to have functional interaction with Sp100.5,6 Therefore, we examined the interaction between E2FBP1 and either Sp100 or UbcH9 in human cells. To check the interaction in vivo, we employed human epidermal carcinoma KB cells and human primary fibroblast TIG-3 cells. First, we tried to detect the interaction between influenza hemagglutinin (HA)-tagged E2FBP1 (HAE2FBP1) and endogenous E2F family proteins. In KB cells transiently transfected with the HA-E2FBP1 expression plasmid, apparent interaction between HA-E2FBP1 and either E2F2 or E2F4 was detected after $20 \mathrm{~h}$ of cultures, although not with other E2F family members including E2F1 (Figure 1a). The result implied that E2FBP1 changes its partner according to the lineage and/or condition of the cells. Next, we transfected $\mathrm{KB}$ cells with the expression plasmid for $\mathrm{HA}$ E2FBP1 together with that for FLAG-tagged UbcH9 (FLAGUbcH9) or FLAG-tagged Sp100 (FLAG-Sp100). Immunoprecipitation of HA-E2FBP1 showed significant interaction with both FLAG-UbcH9 and FLAG-Sp100 (Figure 1b). HAE2FBP1 also interacted with endogenous PML (Figure 1c), although it was not clear whether the interaction was direct or not.

To confirm the interaction between endogenous E2FBP1 (endo-E2FBP1) and either component of PML-NBs, we purified anti-E2FBP1 polyclonal antibody ${ }^{1}$ on an affinity column coupled with bacterially expressed hexagonal histidine-tagged E2FBP1 (rE2FBP1). The specificity of the antibody was checked by immunoblotting against lysates prepared from TIG-3 cells transfected with an empty vector or either expression plasmid for HA-E2FBP1 or E2FBP1 tagged with enhanced green fluorescent protein (E2FBP1G), respectively (Figure 1d). The endo-E2FBP1 was detected as a doublet slightly above and below $75 \mathrm{kDa}$ (closed arrow), and it is presumed as its monomer. The split band is likely to be a result of post-translational modification of E2FBP1, since it contains several target sites for phosphorylation. ${ }^{1}$ The endoE2FBP1 in the lysate that transfected with the empty vector also appeared as an altered form at $100 \mathrm{kDa}$ (open arrow). HA-E2FBP1 and E2FBP1G in the transfected cell lysate (Figure 1d), and nonpurified rE2FBP1 expressed in E. coli at low concentration (data not shown), were only detected as monomers, respectively, while highly purified and concentrated stock of rE2FBP1 appeared preferentially as an altered band at $100 \mathrm{kDa}$ (Figure $3 \mathrm{a}$ ). The result suggests that E2FBP1 is apt to form a tightly bound aggregate; besides, the specificity of the antibody was confirmed. Using this affinitypurified anti-E2FBP1 antibody for both immunoprecipitation and immunoblotting against TIG-3 cell lysate, monomeric endo-E2FBP1 was hardly detectable (Figure 1e, lane 2, lower panel), although it was detected at a slightly higher position than $75 \mathrm{kDa}$ in the immune complex (IP) prepared with antiPML monoclonal antibody (PG-M3) (lane 3, lower panel). The anti-E2FBP1 antibody probably closed the intermolecular distance of endo-E2FBP1 to form an aggregate in the IP, while the altered form of E2FBP1 could not be apparent because of the nonspecific background on the blot (Figure 1e, lane 2). On the other hand, the IP prepared with either anti-E2FBP1 antibody or PG-M3 clearly contained endogenous PML (Figure 1e, lanes 2 and 3 in the middle panel). Considering its molecular size, PML involved in the IP (Figure 1e, open arrow) was assumed to be sumoylated, while the nonsumoylated monomer was only detected in the fraction collected with the anti-E2FBP1 antibody (closed arrow). Sp100 associated with endo-E2FBP1 was detected as a faint but significant band. These findings strongly suggest that E2FBP1 resides in close proximity to, or directly in contact with, PML and Sp100 in vivo. Moreover, endogenous interaction of E2FBP1 and p53 was clearly detected (Figure 1f). Interestingly, E2FBP1-IP contained a greater amount of the altered form of p53 whose molecular weight was shifted at around $12 \mathrm{kDa}$ higher than p53 involved in the p53-IP. The result implied a preferential modification with SUMO-1 adduct when p53 interacted with E2FBP1, since both E2FBP1 and p53 associate with PMLNBs where sumoylated p53 is found preferentially. ${ }^{20}$ The endogenous interaction between E2FBP1 and UbcH9 could not be verified due to interference from the nonspecific background (data not shown).

\section{E2FBP1 localizes in nuclear foci reciprocally with PML}

Since immunoprecipitation analysis showed that E2FBP1 interacts with several components of PML-NBs, it was likely

Figure 1 Interaction of E2FBP1 with Sp100, UbcH9, PML and p53 as well as with E2F2 and E2F4 in vivo. (a) HA-E2FBP1 interacted with E2F2 and E2F4 but not with E2F1 in KB cells transfected with either empty $(-)$ or HA-E2FBP1 expression plasmid $(+)$. IP, immunoprecipitation; IB, immunoblotting; WCL, whole cell lysate. (b) Interaction of HA-E2FBP1 with FLAG-UbcH9 (closed arrow in the upper panel) and FLAG-Sp100 (open arrow) in KB cells. (c) Interaction of HA-E2FBP1 and endogenous PML in KB cells. The identity of the antibody applied is given in parentheses if necessary. (d) Expression of endogenous (endo)-, HA-tagged and GFPtagged E2FBP1 in TIG-3 cells. In all, $10 \mu \mathrm{g}$ of WCL was prepared from TIG-3 cells that were transfected with expression plasmid for the indicated form of recombinant E2FBP1, and subjected to immunoblotting with the indicated antibodies. Closed arrow, monomeric endo-E2FBP1 detected as a doublet; red arrow, HA-tagged E2FBP1 (HA-E2FBP1); green arrow, GFP-tagged E2FBP1 (E2FBP1G); open arrow, altered form of endo-E2FBP1. (e) Endo-E2FBP1 interacted with both endogenous Sp100 and PML in TIG-3 cells. Immune complexes were prepared from equivalent aliquots of TIG-3 cell lysate with the indicated antibodies, and subjected to immunoblotting for the stated antigens, respectively. NRS, pre-immunized rabbit serum. The closed arrow shows the position of the unmodified monomer of each protein, and the open arrow, the position of the sumoylated PML monomer. (f) Endo-E2FBP1 interacted with both sumoylated (open arrow) and nonsumoylated (closed arrow) p53. (g) E2FBP1G (green) partly colocalized with Sp100 (red) as nuclear dots in TIG-3 cells. TIG-3 cells were transfected with expression plasmid for E2FBP1G, stained with anti-Sp100 antibody, and subjected to confocal microscopy. The merged image shows the superimposition for both antigens. Nuclear DNA was visualized with Hoechst (blue). Bar, $10 \mu \mathrm{m}$. (h) Localization of endo-E2FBP1 and PML in TIG-3 cells. Endo-E2FBP1 (red) and PML (green) were stained with the corresponding antibodies. Bar, $10 \mu \mathrm{m}$ 
that E2FBP1 colocalizes with PML-NBs in vivo. We thus checked the colocalization of exogenously transfected E2FBP1G and Sp100 in TIG-3 cells. E2FBP1G appeared throughout the nucleus and colocalized with endogenous Sp100 at nuclear foci (Figure 1g). However, on further analysis, we found that endo-E2FBP1 appeared as ubiquitously stained aggregated speckles and localized in nuclei with PML, but in a reciprocal manner (Figure 1h). To check this finding, the localization of E2FBP1G and endogenous PML was examined (Figure 6, top panels), and we found that expression of E2FBP1G diminished nuclear dots containing PML (i.e. PML-NBs) evidently. Since one report described that Sp100 was retained in nuclear foci after the removal of $\mathrm{PML}$ along with the dissolution of PML-NBs, ${ }^{21}$ the discrepancy observed between colocalization of E2FBP1 with Sp100 and reciprocal localization of E2FBP1 with PML was probably caused by the difference in features of Sp100 and PML. Hence, our findings suggested that E2FBP1 localizes in shells for PML-NBs reciprocally with PML.

\section{E2FBP1 breaks down PML-NBs to liberate their components in vivo and in vitro}

To clarify the functional connection between E2FBP1 and PML-NBs, firstly, we transfected KB cells with a HA-E2FBP1 expression plasmid and checked the effect on the status of PML. Immunoblotting for PML in whole-cell lysate prepared from either KB or TIG-3 cells was not successful. It was assumed as the result of low expression of PML, so that PML in the cell lysate was collected as PML-IP with PG-M3 to get the detectable level. The PML-IP was then subjected to immunoblotting. PML in the IP was detected by rabbit anti$\mathrm{PML}$ polyclonal antibody $\mathrm{H} 238$, and appeared as a slowly migrating broad smear rather than any distinct band when the PML-IP was prepared from either nontransfected or mocktransfected cells (Figure 2a, lane 1). Similarly, Sp100 involved in the PML-IP was detected as a slowly migrating smear by immunoblotting with anti-Sp100 antibody (Figure 3d, lane 1). The results were reproducible, and the indistinct appearance of antigens was inferred from the following evidence that it was a result of tight integration of the PML-IP: (1) previous immunoblotting suggested that the PML in the IP was sumoylated (Figure 1e); (2) Sp100 requires the sumoylation of PML to form a complex (i.e. PML-NBs); ${ }^{22,23}$ (3) sumoylated PML forms a tightly bound aggregate that is little affected by boiling with Laemmli's sample buffer, and sumoylated PML is thereby detected as multiple slowly migrating bands. ${ }^{24}$ The observations also suggested that the PML-IP involved PMLNBs since it contained Sp100. ${ }^{9}$ The discrepancy in the results obtained with $\mathrm{H} 238$ and PG-M3 was probably due to the different epitopes for these antibodies. Employing $\mathrm{H}-238$ for immunoblotting, we found that the expression of HA-E2FBP1 reduced highly integrated forms, but increased the $100 \mathrm{kDa}$ form of PML (Figure 2a, lane 2). The results suggest that overexpression of E2FBP1 causes the disintegration of PML complex (i.e. PML-NBs) by releasing PML in vivo.

We further checked the effect of E2FBP1 overexpression on the status of $\mathrm{p} 53$, since previous data suggested that endoE2FBP1 bound preferentially with a slowly migrating form of

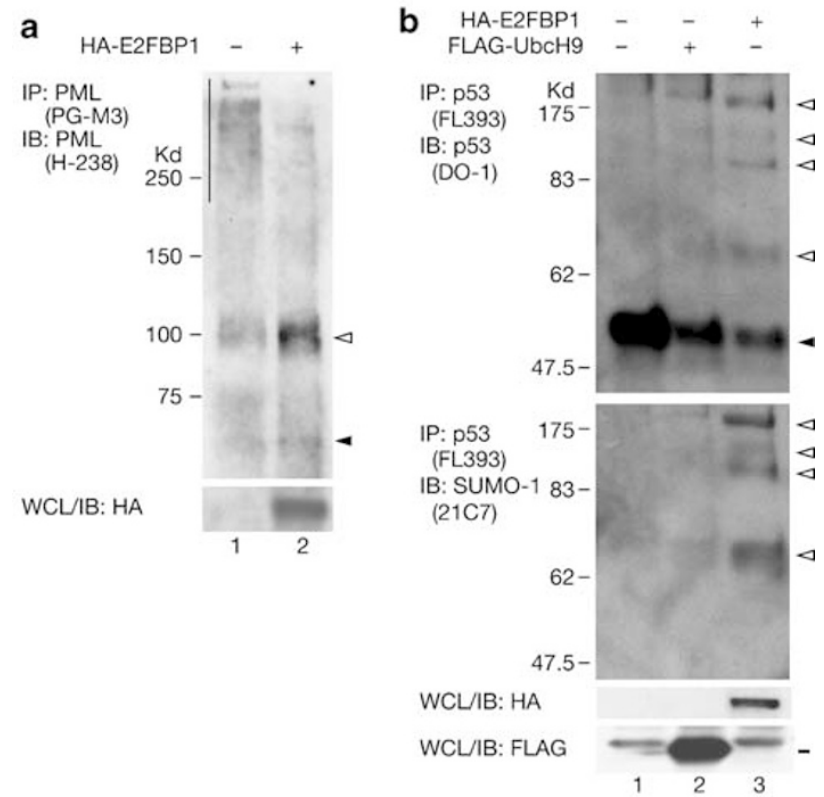

Figure 2 Expression of HA-E2FBP1 induced both convergence of the high molecular weight PML complex to a $100 \mathrm{kDa}$ form and a proportional increase in sumoylated p53 complex in vivo. (a) PML was immunoprecipitated from KB cells transfected with empty (-) or HA-E2FBP1 expression plasmid, and detected by immunoblotting with the indicated antibodies. The closed arrow shows the position of the nonsumoylated PML monomer. The open arrow shows the $100 \mathrm{kDa}$ form of PML. The line indicates the high molecular weight complex containing PML. The corresponding WCL was subjected to immunoblotting for HA. (b) p53 was immunoprecipitated from KB cells transfected with empty vector or expression plasmid for the indicated factor, and detected on immunoblots using the antibodies shown. The closed arrow shows the position of the free form of p53. Open arrows indicate the positions of sumoylated and oligomerized forms of p53. The corresponding WCL was subjected to immunoblotting for HA and FLAG

p53 that seemed to be sumoylated (Figure 1f). Immunoprecipitation for p53 revealed that expression of HA-E2FBP1 resulted in a marked increase of the slowly migrating forms of p53, and subsequent analysis showed that the modified molecules contained SUMO-1 adduct (Figure 2b, lane 3, open arrows), whereas the original band for p53 was relatively decreased (closed arrow). Since the sumoylation of p53 was reported to occur at a single site ${ }^{25,26}$ and the positions of the bands were mostly consistent with multiples of sumoylated p53, the slowly migrating forms (upper three bands) were presumed to be oligomers. The efficiency of the sumoylation and oligomerization of p53 induced by HA-E2FBP1 was much greater than that induced by FLAG-UbcH9 (Figure 2b, lane 2).

Since sumoylated p53 was preferentially expressed in PML-NBs, ${ }^{20}$ the induction of $\mathrm{p} 53$ sumoylation could be a result of the liberation of sumoylated p53 from PML-NBs by its disintegration. To verify this hypothesis, we checked whether E2FBP1 disintegrates PML complex to release sumoylated p53 in vitro. PML complex prepared as PML-IP from $5 \times 10^{6}$ TIG-3 cells per reaction was incubated with or without purified rE2FBP1 (Figure 3b-d). The reaction mixture was then subjected to immunoblotting. As a control, an aliquot of the PML-IP was incubated with a GST-tagged yeast desumoylating enzyme, Ulp $1^{27}$ (Figure 3b, lanes 5 and 10, top panels). GST-Ulp1 caused disappearance of slowly migrating forms of 


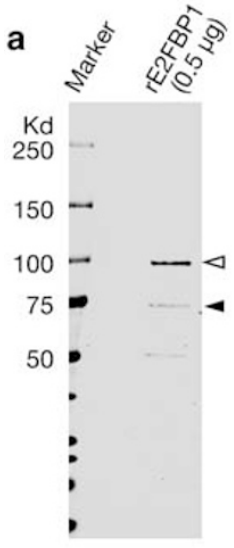

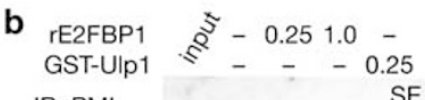
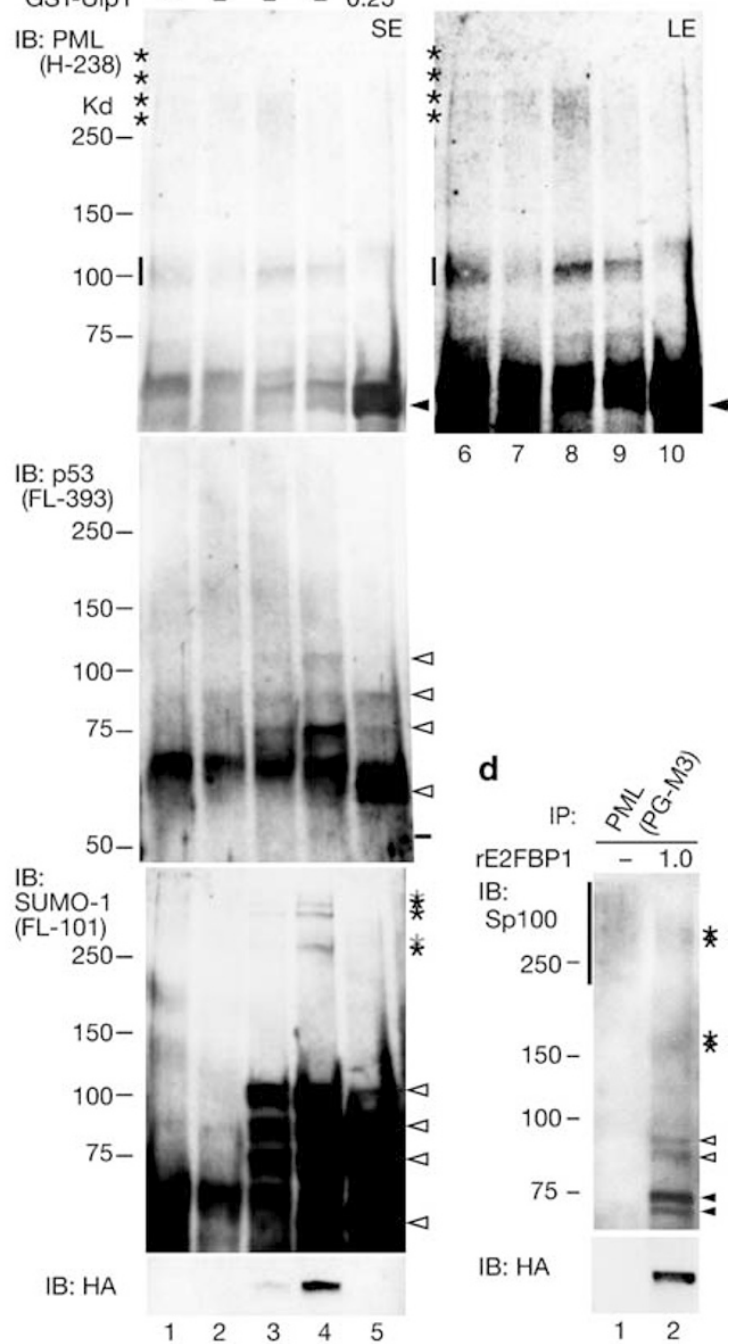

Figure 3 E2FBP1 disintegrated PML complex and liberated PML and protein complexes containing p53 and/or Sp100 in vitro. (a) The purity of the bacterially prepared E2FBP1 (rE2FBP1) was confirmed by staining. Closed and open arrows show the positions of the monomer and altered form of rE2FBP1, respectively. (b) E2FBP1 disintegrated the PML complex. An aliquot of IP of PML prepared from TIG-3 cell lysate was incubated with the factor mentioned above, and subsequently subjected to immunoblotting with the indicated antibodies. The upper two panels represent images of an identical immunoblot exposed to X-ray film briefly (SE) and long time (LE), respectively. Closed arrows show the position of the nonsumoylated monomer of PML. Asterisks show positions of high molecular weight complexes containing PML. A short line shows the position of the $100 \mathrm{kDa}$ form of PML complex. Open arrows show positions of sumoylated protein complexes containing p53. Horizontal line indicates the position of p53 monomer. (c) E2FBP1 liberated and disclosed sumoylated forms of p53 in both the p53-IP and the PML-IP. An aliquot of IP of p53 and PML prepared from TIG-3 cell lysate was incubated with or without $1.0 \mu \mathrm{g}$ of E2FBP1, and subsequently subjected to immunoblotting with the indicated antibodies. Open arrows show positions of the sumoylated p53 and its complex. The closed arrow shows the position of the nonsumoylated monomer of p53. (d) E2FBP1 liberated Sp100 as well as PML and p53 from the PML-IP. An aliquot of the PML-IP prepared from TIG-3 cell lysate was incubated with or without $1.0 \mu \mathrm{g}$ of E2FBP1, and subjected to immunoblotting with anti-Sp100 antibody. The vertical line shows distribution of Sp100 involved in the high molecular weight PML complex. Open and closed arrows indicate the positions of sumoylated and nonsumoylated Sp100. Asterisks show positions of the sumoylated Sp100 complexes

the PML complex (positions are indicated by asterisks in Figure 3b, lane 1, upper panels), but it caused accumulation of the nonsumoylated PML monomer (lane 5, closed arrow) as well as distinct bands containing p53 (p53 complexes) (open arrows). Compared with the integrated PML complex (lanes 1 and 2), nonsumoylated PML monomer and released p53 complexes emerged much intensively (lane 5). It is probably caused by disclosure of epitopes along with the disintegration of PML complex. rE2FBP1 also liberated the nonsumoylated
PML monomer and p53 complexes in a dose-dependent manner (compare lanes 2, 3and 4). Interestingly, while liberated PML was focused in a band of nonmodified form by $\mathrm{rE2FBP1}$, the number of liberated p53 complexes was rather increased in higher molecular weight positions along with the amount of rE2FBP1. The positions of $p 53$ complexes revealed to be consistent with those shown by the anti-SUMO1 antibody (Figure 3b, lower panel, open arrows). The result was reproduced in a similar assay using p53-IP collected with 
the anti-p53 antibody DO-1 (Figure 3c, lanes 1 and 2). After incubation with rE2FBP1, the p53-IP released both sumoylated (open arrows) and nonsumoylated forms (closed arrow) of $p 53$. The liberated forms of p53 showed identical positions with that released from the PML-IP (compare lanes 2 and 4 in the upper and middle panels). In addition to p53, Sp100 in the PML-IP was also released in both sumoylated and nonsumoylated forms after incubation with rE2FBP1 (Figure 3d). Hence, the proportional increase of sumoylated p53 complex in the HA-E2FBP1 expression cells shown in Figure $2 b$ was most probably a result of disintegration of PML-NBs and consequent release of sumoylated p53 complexes. Taken together, E2FBP1 is most likely to induce specific desumoylation of PML to disintegrate PML-NBs, and thereby sumoylated protein complexes including both Sp100 and tightly bound sumoylated p53 complexes are liberated from the PML complex (i.e. PML-NBs) in vivo and in vitro.

\section{E2FBP1 disperses PML-NBs as small speckles in vivo}

The results obtained by in vitro analysis strongly suggested that E2FBP1 induces the disintegration rather than proteolytic degradation of PML-NBs to release their components, which seems to be consistent with the results obtained for the transient expression analysis (Figure 2) and immunofluorescence confocal microscopy (Figure $1 \mathrm{~g}$ and $\mathrm{h}$ ). To check how E2FBP1 breaks down PML-NBs, nontagged wild-type E2FBP1 cDNA linked to the mouse mammary tumor virus (MMTV) LTR promoter was introduced into TIG-3 cells at 34 population doublings (PD), and clones expressing E2FBP1 in response to dexamethasone (dex) were isolated. Since the clones were near the end of their lifespan, it was difficult to prepare enough amount of cell lysate for immunoprecipitation analysis. Therefore, only expression levels of E2FBP1 and Sp100 were checked by immunoblotting. Among the clones, TIG-E7 cells expressed E2FBP1 most efficiently in response to $1 \mu \mathrm{M}$ dex (Figure $4 \mathrm{a}$, upper). Curiously, the majority of overexpressed E2FBP1 was at a higher position in the doublet of the monomer (open arrow), while the majority of the constitutively expressed endo-E2FBP1 was at a lower position (closed arrow). The difference in molecular size of E2FBP1 is likely to reflect its phosphorylation status since the protein contains multiple phosphorylation target motifs, ${ }^{1}$ albeit the significance of its phosphorylation is unclear. The expression level of Sp100 in TIG-E7 cells was decreased along with the ectopic expression of E2FBP1, while Sp100 expression in other clones that were not induced by E2FBP1 showed no significant change in response to dex (Figure $4 \mathrm{a}$, lower).

Although the biochemical analysis was not enough, the effect of ectopically expressed E2FBP1 was nevertheless observed by immunofluorescence confocal microscopy. The result revealed that the cells of isolated clones showed hyperplasis of PML-NBs and indistinct expression of E2FBP1 in the absence of dex (Figure 4b, panels 1 and 3), whereas TIG-E7 cells expressed E2FBP1 distinctly in the nucleus and PML-NBs were scattered in the cytoplasm as small speckles in the presence of dex (Figure 4b, panel 4). TIG-neo cells a

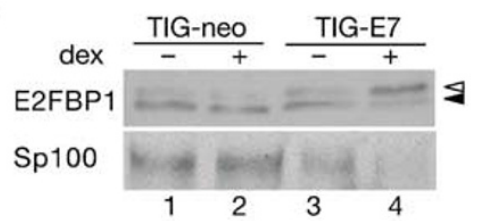

b

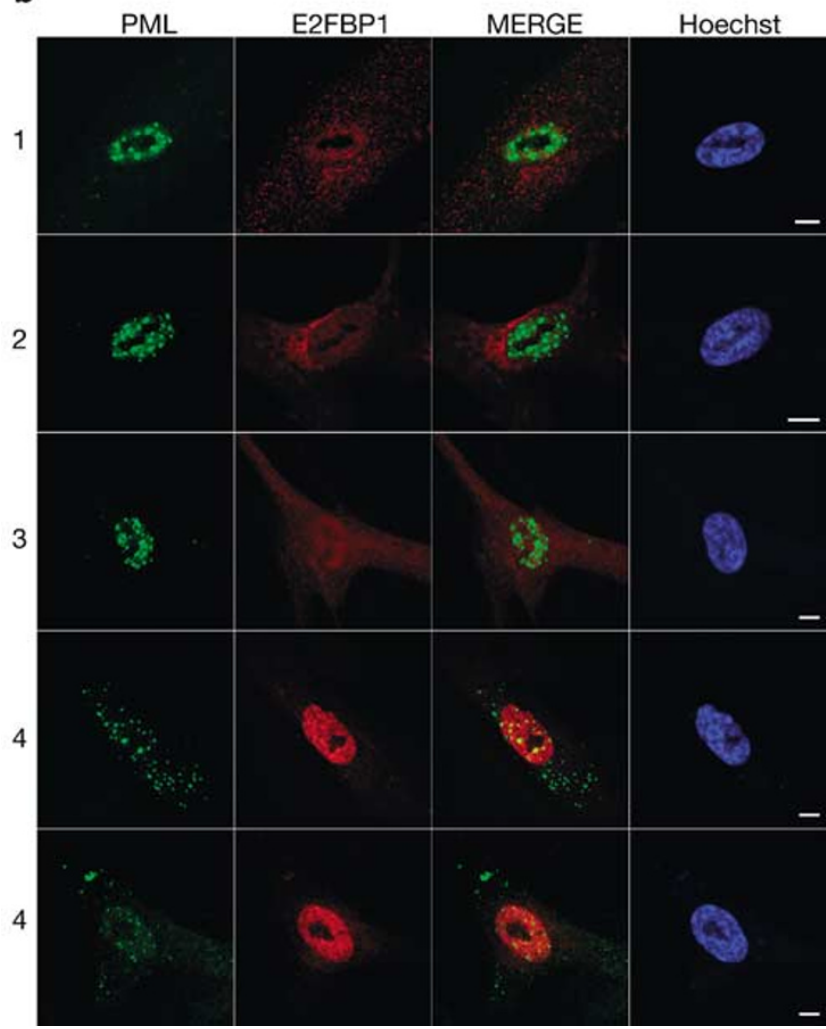

Figure 4 Overexpression of E2FBP1 disperses PML-NBs as small speckles in aged TIG-3 cells. (a) TIG-3 cells at 36 PD were transfected with pMTV-E2FBP1 to obtain clones overexpressing WT E2FBP1 in response to dex. The isolated clone TIG-E7 and control clone TIG-neo obtained by introduction of pSV2-neo were cultured with or without $1 \mu \mathrm{M}$ dex for $24 \mathrm{~h}$, and subsequently the cells were lysed and subjected to immunoblotting with either anti-E2FBP1 antibody or antiSp100 antibody. (b) Another aliquot of the cells was stained with indicated antibodies, and then observed by confocal immunofluorescent microscope. Numbers on the left of the panels indicate that those cells shown in the panels correspond to the cell lysate subjected to immunoblotting. Bar, $10 \mu \mathrm{m}$

showed no obvious changes after treatment with dex (panels 2). Taken together with previous results, these observations suggest that E2FBP1 induces the disintegration of PML-NBs and subsequent degradation of the component of PML-NBs such as Sp100.

\section{E2FBP1 requires acidic $\mathrm{N}$-terminal portion for efficient nuclear localization, and requires ARID for the disintegration of PML-NBs}

To elucidate the connection between the localization and function of E2FBP1, we constructed several deletion mutants of E2FBP1G (Figure 5c) and transfected them into TIG-3 cells for microscopic analysis (Figure 6). Consistent with the results shown above, wild-type (WT) E2FBP1G showed an aptitude 


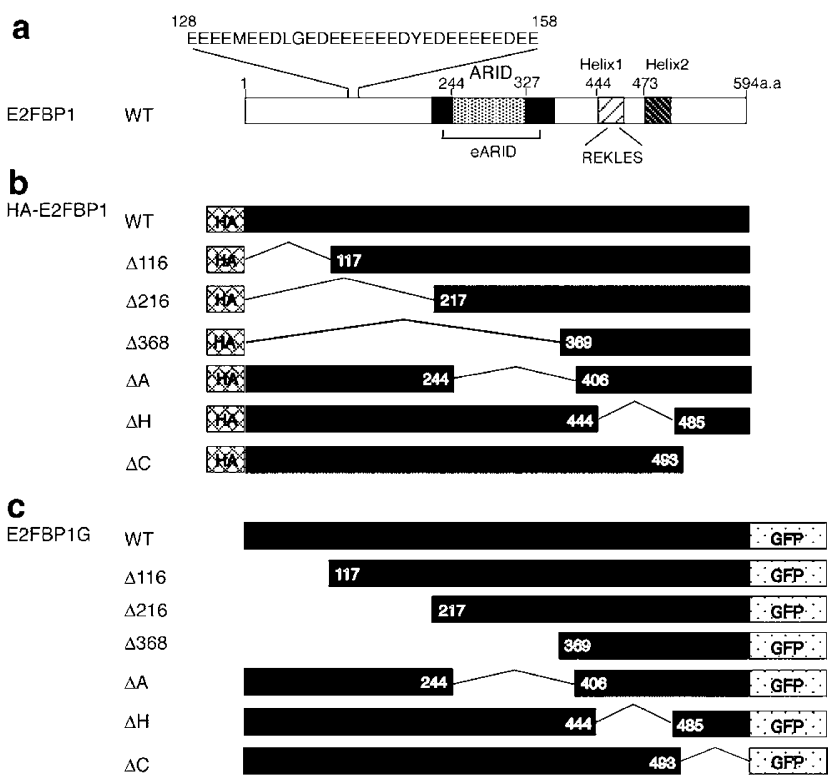

Figure 5 E2FBP1 and its deletion mutants for transient expression assay. (a) Full-length (WT) E2FBP1 cDNA possesses E-rich, eARID and HLH domains. A 'REKLES' motif is encoded in Helix-1 of the HLH-domain. (b) HA-E2FBP1 and its deletion mutants. (c) E2FBP1G and its deletion mutants

to localize in nuclei and diminished PML-NBs. The N-terminal deletion mutant $\Delta 116$ appeared clearly in the nucleus and abolished PML-NBs completely. However, further deletion in the N-terminal region of $\Delta 216$ mutant removed E2FBP1G to the cytoplasmic meshwork which developed at the fringe of the nucleus, and seemed to cause the hyperplasis of PMLNBs. Thus, the N-terminal acidic portion between amino acids (aa) 117 and 216, which includes an E-rich region (Figure 5a), is required for efficient nuclear localization of E2FBP1 and, consequently, for diminishing PML-NBs. The most N-terminal region between 1 and 116 aa may regulate the function of the acidic portion. Interestingly, deletion of the $\Delta 368$ mutant restored the localization of E2FBP1 to the nucleus but eliminated the function of diminishing PML-NBs. Other mutants lacking the ARID (i.e. $\Delta \mathrm{A}$ ) exhibited a similar localization to WT, but showed no effect on PML-NBs. On the other hand, the $\Delta \mathrm{H}$ and $\Delta \mathrm{C}$ mutants retained the function of diminishing PML-NBs. Thus, from the results shown in Figures 1h and 4, E2FBP1 disrupts PML-NBs via a function of ARID when it resides in the nucleus at high levels. The data also suggest that the $\mathrm{N}$-terminal acidic portion directs the nuclear localization of E2FBP1. Similar results were obtained with another set of E2FBP1 deletion mutants tagged with $\mathrm{HA}$ at the $\mathrm{N}$-terminus (Figure 5b, data not shown).

\section{Downregulation of E2FBP1 induces growth suppression and premature senescence by inducing hyperplasis of PML-NBs}

To elucidate the role of endo-E2FBP1 in the cellular controls, we prepared anti-E2FBP1 short interfering (si) RNA (siRNAE) (for review, see Hannon ${ }^{28}$ ) (Figure 7a). A BLAST search revealed that no more than 17 base pairs (bps) among the 19bp core sequence of siRNA-E were homologous with any registered DNA sequence. A nonsense control siRNA (siRNA$\mathrm{N}$ ) that showed no significant (i.e. more than $17 \mathrm{bps}$ among the 19-bp core sequence) homology with any mammalian gene was also designed for minimum disturbance of cellular gene expression.

The effect of downregulation of E2FBP1 was first observed on the growth of actively proliferating TIG-3 cells. The cells were repeatedly treated with either of the siRNA after every passage from 36 PD (Figure 7b). After the third passage, siRNA-E treatment markedly suppressed cell growth, while siRNA-N treatment had only a slight effect (Figure $7 \mathrm{~b}$ ). To connect the effect to the expression levels of several cell cycle-regulating factors, aliquots of the cells were subjected to immunoblotting after 2 days of the third treatment (Figure 7c). The result showed significant increases in the expression levels of $\mathrm{p} 16^{\mathrm{INK} 4 \mathrm{~A}}, \mathrm{p} 53, \mathrm{PAl}-1$ and Sp100; in contrast to this, the expression level of E2FBP1 was apparently decreased. Although no changes in other factors including p21 WAF1/CIP1 were observed, the growth suppression linked to upregulation of PAl-1, p16 ${ }^{\text {INK4A }}$ and p53 suggested the induction of premature senescence. ${ }^{18,29,30}$ To test this possibility, aliquots of the cells were subjected to a SA- $\beta$-gal assay ${ }^{30,31}$ after 2 days of the fourth treatment (Figure 7d). Consistent with the results of immunoblotting stated above, only the cells treated with siRNA-E showed an apparent population expressing SA$\beta$-gal. Thus, the induction of premature senescence by the downregulation of E2FBP1 was evident.

To check whether downregulation of E2FBP1 induced features of premature senescence through the function of PML-NBs, aliquots of TIG-3 cells were treated with both SiRNA-E and anti-PML siRNA (siRNA-P) ${ }^{32}$ after every passage from $36 \mathrm{PD}$. In contrast to the cells treated only with siRNA-E, the cells treated with both siRNA-E and siRNA-P did not show significant growth arrest (Figure 7e). The result clearly showed that downregulation of PML rescued siRNA-Einduced cell cycle arrest, and thus suggested that downregulation of E2FBP1 induced premature senescence through the function of PML. Confocal microscopy connected these observations to the status of PML-NBs. The siRNA-Emediated downregulation of E2FBP1 resulted in depletion of E2FBP1 from nuclei and an obvious upregulation of NB formation (i.e. hyperplasis of PML-NBs) (Figure 8E). siRNA-N treatment of the cells produced no apparent alteration in the level and localization of E2FBP1 and PML-NBs (compared with untreated cells, shown in Figure $1 \mathrm{~h}$ ). In contrast, siRNA-P suppressed the formation of PML-NBs nearly completely even with the downregulation of E2FBP1 (Figure 8, P and $P+E)$. These results strongly suggest that downregulation of E2FBP1 induces hyperplasis of PML-NBs and, consequently, cells are committed to premature senescence by the function of PML-NBs.

\section{Discussion}

In this report, we showed that E2FBP1 functions as a negative regulator of PML-NBs. The finding that E2FBP1 binds endogenously to multiple components of PML-NBs including 


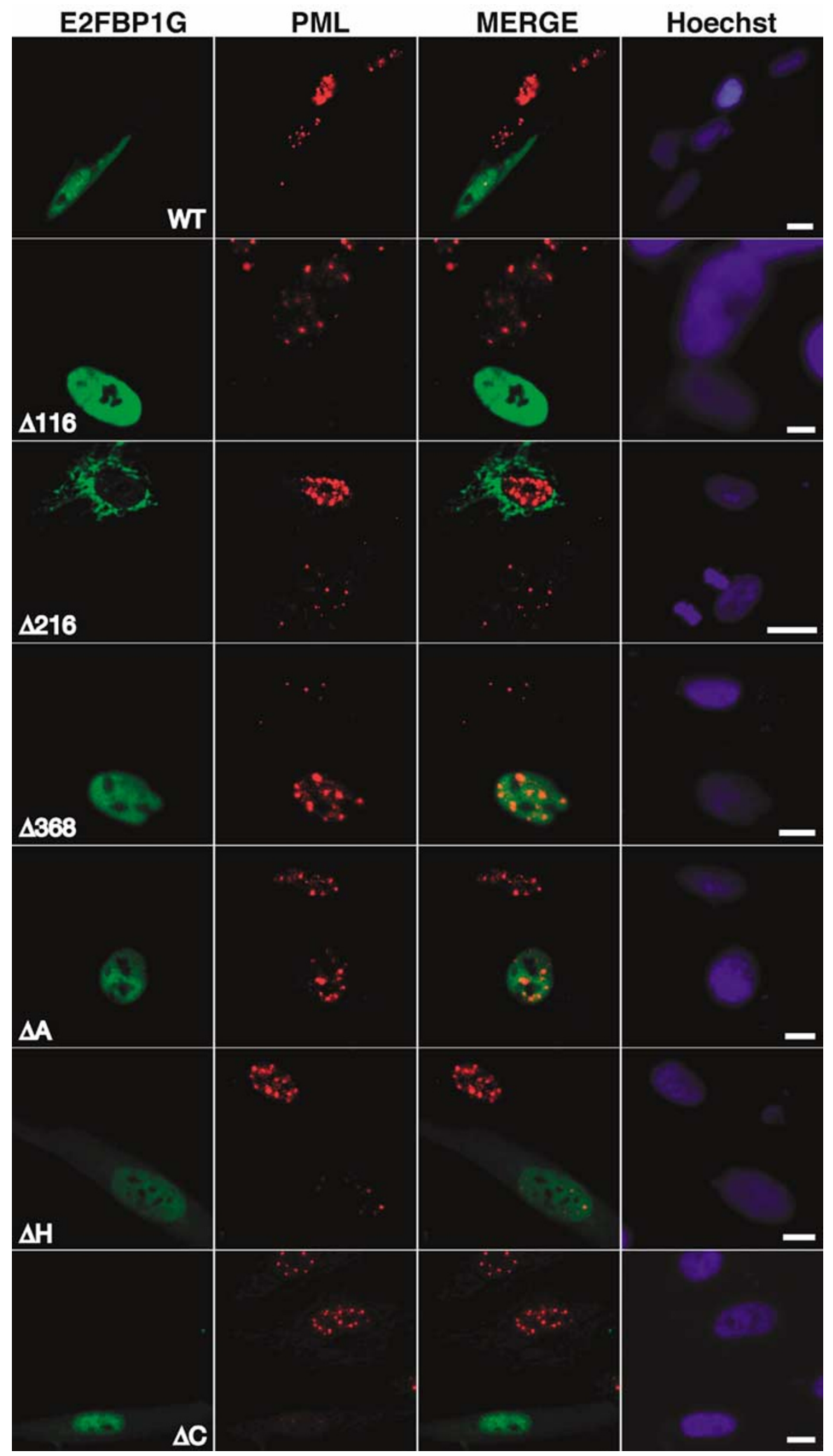

Figure 6 E2FBP1 diminished PML-NBs through nuclear localization and ARID. TIG-3 cells at 42 PD were transfected with the expression plasmid for E2FBP1G deletion mutants (green) and cultured for $40 \mathrm{~h}$. Subsequently, the cells were stained with anti-PML antibody (PG-M3, red) and Hoechst 33342 dye (blue), and subjected to confocal immunofluorescent microscopy. Bar, $10 \mu \mathrm{m}$ 
a

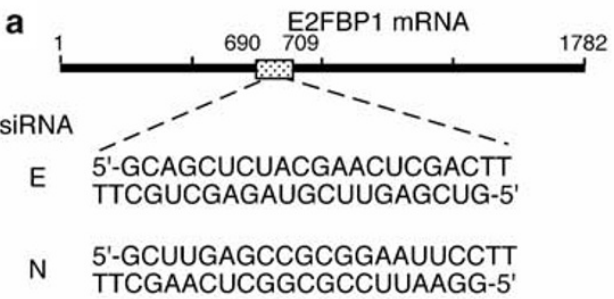

b

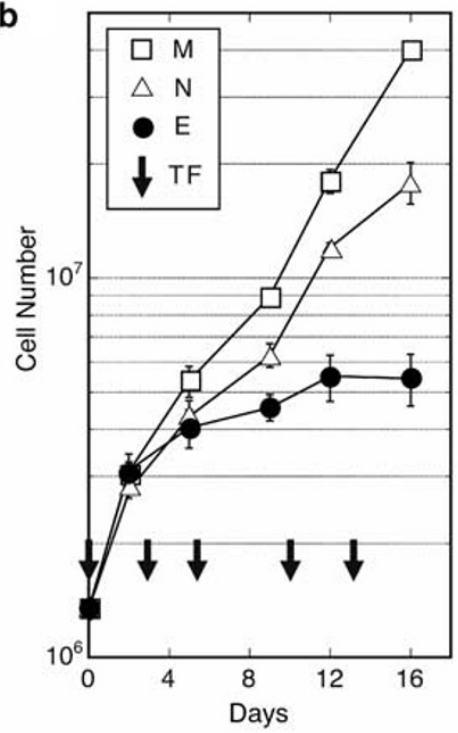

C

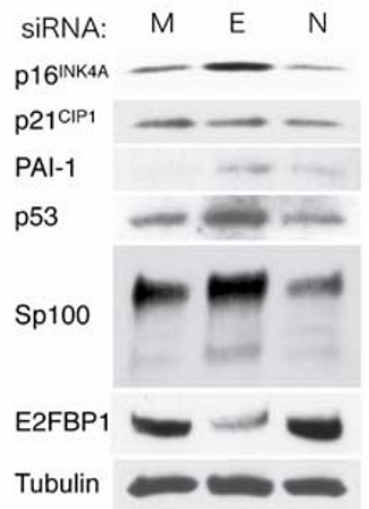

e d
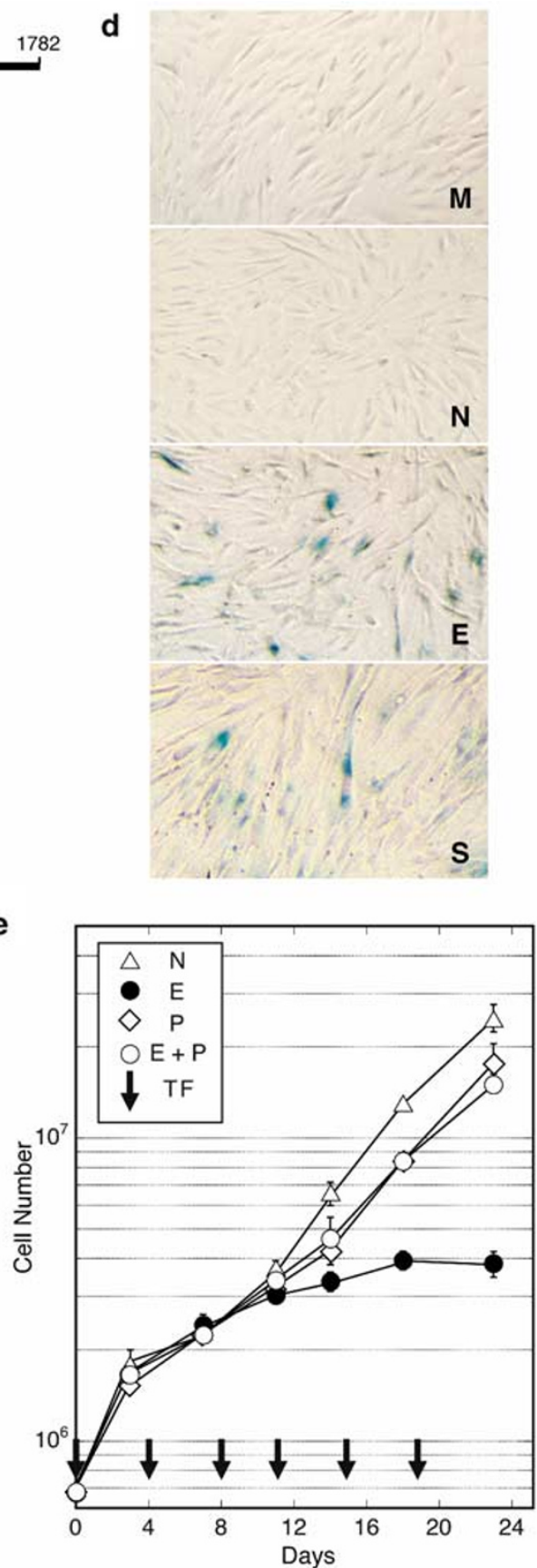

Figure 7 Downregulation of E2FBP1 committed human primary fibroblast cells to premature senescence. (a) Sequences of siRNA-E (E) and siRNA-N (N). The top line represents the ORF of E2FBP1, and the hatched box represents the position of the target sequence of siRNA-E. (b) Downregulation of E2FBP1 resulted in a suppression of cell growth. TIG-3 cells at 36 PD were treated with either of siRNA or mock reagent after every passage (arrows) and counted. The values and error bars represent averages and standard deviations of three independent assays. (c) Induction of senescent marker proteins by downregulation of E2FBP1. WCLs were prepared from the cells after 2 days of the third passage, and were subjected to immunoblotting for the indicated antigens. (d) Induction of SA- $\beta$-gal expression as a result of downregulation of E2FBP1. The activity of SA- $\beta$-gal in the cells treated with siRNAs was checked after 2 days of the fourth passage. Cells at 57 PD (S) were stained for comparison. (e) Simultaneous downregulation of PML rescued TIG-3 cells from growth arrest induced by the downregulation of E2FBP1. TIG-3 cells at 36 PD were treated with the indicated siRNA or mock reagent after every passage (arrows) and counted. The values and error bars represent averages and standard deviations of three independent assays

PML, Sp100 and p53 (Figure 1e and f) implied that E2FBP1 could be acting as an adaptor protein, for example, like Daxx, to link these components. ${ }^{23}$ However, the nuclear localization of E2FBP1 was inconsistent with that of PML (Figure 1h). To elucidate the functional connection between E2FBP1 and PML-NBs, the effect of E2FBP1 on immunologically prepared PML-complexes (i.e. PML-IP containing PML-NBs) was analyzed in vivo (Figure 2) and in vitro (Figure $3 b-d)$. The 


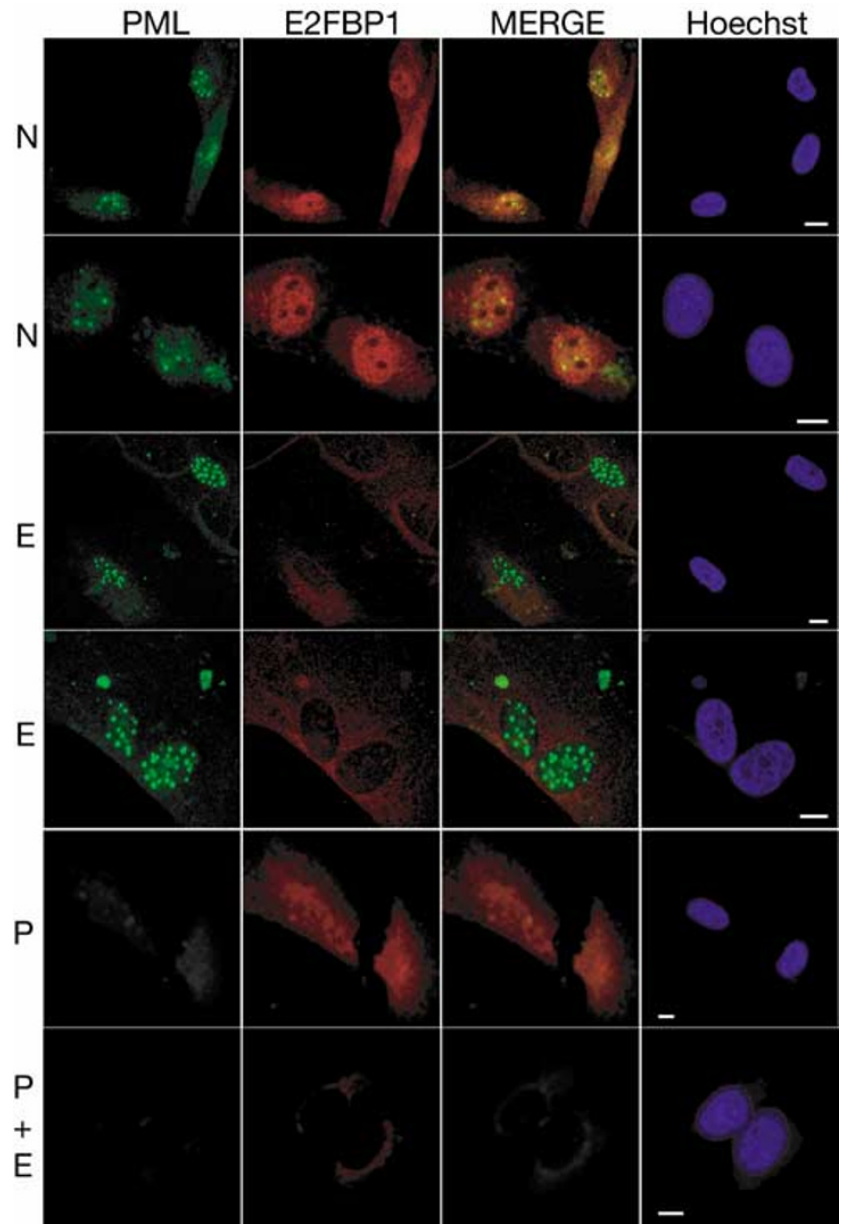

Figure 8 Enhanced development of PML-NBs and overexpression of $p 53$ were induced by the downregulation of E2FBP1. TIG-3 cells at 36 PD were similarly treated with either of the siRNA, as shown in Figure $7 \mathrm{~b}$ and e. At 2 days after the third passage, the cells were subjected to immunostaining for E2FBP1 (red), DNA (blue) and PML, and subsequently observed by confocal microscope. In panels, both lower (row 1 and 3 ) and higher (row 2 and 4) magnification images are shown. Bar, $10 \mu \mathrm{m}$

result revealed that PML in the PML-IP prepared with the antiPML monoclonal antibody PG-M3 was detected as faint bands by the anti-PML polyclonal antibody H238 (Figures 2a, lane 1; 3b, lane 1). Both Sp100 and p53 involved in the PML-IP also appeared as indistinct smears with the respective antibodies (Figures 3b, lane 1; 3c, lane 3; 3d, lane 1). To better elucidate the components, the PML-IP was treated with the denaturing reagents such as $1 \%$ SDS plus $1 \%$ DOC, $2 \mathrm{M}$ guanidine hydrochloride, $8 \mathrm{M}$ urea or $3 \mathrm{M}$ thiocyanate ${ }^{33}$ (data not shown); however, none of them liberated the component in the complex. In contrast, both E2FBP1 and Ulp1 efficiently broke down the PML complex to disclose clear signals on the blot (Figures 2a, lane 2; 3b, lanes 3-5; 3c, lane 4; 3d, lane 2). Moreover, p53-IP was revealed to contain the same sumoylated components as PML-IP, and those components were also disclosed by E2FBP1. From these results, it is evident that the tightly integrated PML complex containing multiple components of PML-NBs are involved in both the PML- and p53-IP, and that E2FBP1 breaks down this PML complex to liberate the components. Since sumoylation of PML is essential for forming PML-NBs ${ }^{22,23}$ and the E2FBP1mediated disintegration of the PML complex accompanies an increase in the liberation of the nonsumoylated $P M L$ monomer (Figure 3b, top panels), E2FBP1 seems to function to induce the desumoylation of PML. However, in contrast to the desumoylating enzyme Ulp1 that breaks down the PML complex and mainly liberates lower molecular weight components, E2FBP1 does not weaken signals of sumoylated components of higher molecular weight, but rather strengthens them (Figure 3b, third panel). Moreover, in primary structure, E2FBP1 shows significant homology with neither SUMO-1 hydrolases nor SUMO-1 ligases. Therefore, E2FBP1 itself is not likely to be a desumoylating enzyme.

We also demonstrated a negative function of E2FBP1 to PML-NBs in TIG-3 cells by both overexpression and downregulation analyses. Nuclear localization of an excess of E2FBP1 markedly dispersed and diminished PML-NBs (Figure 4). The result was consistent with the conclusion based on biochemical analyses shown in Figures 2 and 3 (i.e. E2FBP1 disintegrates PML-NBs). A domain corresponding to the downregulation of PML-NBs coincided with ARID of E2FBP1 (Figure 6). The ARID has been found in a vast number of DNA-binding factors throughout eukaryotes, ${ }^{3}$ and the closest homologue of E2FBP1, Bright, also binds with PML-NBs and is implicated in the remodeling of the local chromatin structure through binding to the MAR motif. ${ }^{5,34}$ However, the role of ARID for E2FBP1 is not clear since rE2FBP1 disintegrates PML complex in vitro without additional DNA in the reaction, while the necessity of both the nuclear localization and the ARID for diminishing PML-NBs was shown in vivo (Figure 6). In contrast to the results of overexpression analyses, sequestration and/or depletion of E2FBP1 from the nucleus by either the $\Delta 216$ mutant (Figure 6) or siRNA-mediated downregulation (Figure 8) enhanced the development of PML-NBs in TIG-3 cells. Moreover, the analyses of cell growth and expression markers revealed that the downregulation of E2FBP1 induces premature senescence in TIG-3 cells. Since the simultaneous suppression of PML by siRNA-P rescued TIG-3 cells from siRNA-E-induced growth arrest, the downregulation of E2FBP1 induces premature senescence through the function of PML, that is, the formation of PML-NBs (Figure 7). Although our observation is consistent with the report that E2FBP1 rescues oncogenic Ras-induced premature senescence ${ }^{35}$ the molecular mechanism linking the function of E2FBP1, PML-NBs and induction of premature senescence remains unclear.

Finally, a novel mechanism regulating cellular integrity was revealed through analyses of the function of E2FBP1; however, the role of E2FBP1 was only partly elucidated. Since E2FBP1 is a DNA-binding and E2F-binding protein, elucidation of its function should provide more details of the governance system in cells.

\section{Materials and Methods}

\section{Construction of plasmids}

The full-length E2FBP1 cDNA was cloned from a cDNA library of TIG-3 cells using a truncated cDNA cloned from the human embryonal 
carcinoma cell line NEC14 ${ }^{1}$ as a probe, and was amplified by Pfu DNA polymerase (Pfu pol) with the following primers to add Bgll and Xhol sites at the $5^{\prime}$ and $3^{\prime}$ ends, respectively: E2FBP1-s (5'-TGAATAGATCTCCGCAGGGCCATGAAAC-3') and E2FBP1-as 1 (5'-TTCTCGAGGATCCTTTCTGGATATCTTCC-3'). The amplified CDNA was inserted between BamHI and Xhol sites of pcDNA3 (Invitrogen, Carlsbad, CA, USA), and then the CDNA was again cleaved out with Hindlll and BamHI and inserted between Hindlll and Bgll sites of pMTV-dhfr to link E2FBP1 cDNA to mouse mammary tumor virus LTR and construct pMTV-E2FBP1. To add an HA-tag to the N-terminal side of E2FBP1, a duplicated HA-tag sequence was inserted between Hindlll and Kpnl sites, that were $5^{\prime}$ to the cDNA, yielding pcDNA3-HA-E2FBP1. To add a GFP-tag, E2FBP1 cDNA was re-amplified by $P f u$ pol with E2FBP1-s and E2FBP1-as2 (5'GAGTTAGACCGGTGGCAACGAGTTATTTGA-3') to remove the termination codon and to add an Agel site at the $3^{\prime}$ end. The amplified CDNA was then inserted between the Bgll and Agel sites of pd2N1-EGFP (Clontech, Palo Alto, CA, USA) to construct pd2E2FBP1G. To construct deletion mutants of E2FBP1G, cDNA fragments generated by Pfu pol with the primers, $\Delta 116$-s (5'-GCGGTACCATGGCCTCCGACGAGG-3'), $\triangle 216$-s (5'-GCGGTACCATGCAGCCGCCTGACCAC-3'), $\quad$ 368-s (5'-GCGGTACCATGCTCTCCTCACCCAAG-3'), $\triangle$ A-s (5'-CGAATTCCCCATCACAGTCCCTG-3'), $\triangle \mathrm{H}$-as (5'-CGGGTTCGTCGACACCGGTACCTTAAG-3'), $\triangle$ C-as ( $5^{\prime}$-GAGTTAGACCGGTATGCTCATGGGCAGC-3') and E2FBP1-as2, were inserted between $K p n l$ and Agel sites $(\Delta 116-G$, $\triangle 216-\mathrm{G}$ and $\Delta 368-\mathrm{G})$ of pd2EGFP-N1, or used to replace a corresponding region of pd2E2FBP1G between $\mathrm{EcoRl}$ and Agel sites $(\triangle \mathrm{A}-\mathrm{G}, \Delta \mathrm{H}-\mathrm{G}$ and $\Delta \mathrm{C}-\mathrm{G})$. Deletion mutants of $\mathrm{HA}-\mathrm{E} 2 \mathrm{FBP} 1$ were then constructed by replacing the corresponding region of pcDNA3-HAE2FBP1 with Kpnl-Agel fragments of E2FBP1G deletion mutants. For two-hybrid screening, E2FBP1 cDNA was fused in frame to GAL4-DBD in pGBDU ${ }^{19}$ to generate $\mathrm{pGBDU}-\mathrm{E} 2 \mathrm{FBP} 1$. To prepare bacterially expressed recombinant E2FBP1 (rE2FBP1), the HA-E2FBP1 sequence in pcDNA3HA-E2FBP1 was amplified and fused with a hexagonal histidine $\left((\mathrm{His})_{6}\right)-$ tag sequence at the $5^{\prime}$ end by PCR. The amplified (His) $)_{6}-\mathrm{HA}-\mathrm{E} 2 \mathrm{FBP} 1$ fragment was subcloned into pCR2.1 to construct pCR2-(His) $)_{6}$-E2FBP1. To generate an Sp100 expression vector, pAD-GAL4-2.1-Sp100 was cleaved by EcoRI and Sall, and Sp100 cDNA was inserted between the EcoRI and Xhol sites of pcDNA3. A FLAG-tag sequence was then added between HindllI and EcoRI sites, which lie $5^{\prime}$ to the $\mathrm{CDNA}$, to yield pcDNA3-FLAG-Sp100. To construct the UbcH9 expression vector, a CDNA fragment was cleaved from pAD-GAL4-2.1-UbcH9 by EcoRI-Xhol, and inserted into pcDNA3. The FLAG-tag sequence was then added between HindllI and EcoRI sites that lie $5^{\prime}$ to the UbcH9 cDNA, to construct pcDNA3-FLAG-UbcH9.

\section{Cells and transfection}

The human epidermoid carcinoma cell line KB was cultivated in Dulbecco's modified Eagles medium (DME) supplemented with $8 \%$ fetal calf serum (FCS). TIG-3 human fetal lung primary fibroblast cells were obtained from the Japanese Collection of Research Bioresources and cultivated in DME supplemented with 10\% FCS. The cells were used for experiments at PD of between 36 and 50 . For transfection, cells were replated to be $50 \%$ confluent and transfected using FuGene 6 transfection reagent (Roche, Basel, Switzerland) for $44 \mathrm{~h}$ according to the manufacturer's directions. To analyze the effect of overexpression of WT E2FBP1, TIG-3 cells at 34 PD were transfected with pSV2-neo and/or pMTV-E2FBP1. The cells were then cultivated in the presence of $720 \mu \mathrm{M}$ of G418 for 2 weeks and subsequently in the presence of $250 \mu \mathrm{M}$ of G418 for 3 weeks to develop colonies. The colonies were isolated and a few clones gave rise to enough cells for further analyses although the majority of clones were near the end of their lifespan.

\section{Yeast two-hybrid screening}

A human liver cDNA library for two-hybrid screening was purchased from Stratagene. Saccharomyces cerevisiae strain PJ64-9A was transformed with the cDNA library and pGBDU-E2FBP1, and screened on a synthetic medium (0.67\% Bacto-yeast nitrogen base, $2 \%$ glucose and $20 \mu \mathrm{g}$ each/ $\mathrm{ml}$ of the amino acids tryptophan, lysine and methionine). The cDNA clones obtained were further subjected to reproducible co-transformation with PGBDU-E2FBP1 on the synthetic medium.

\section{Immunoprecipitation and immunoblotting}

To detect the transient expression of tagged proteins, transfected cells were washed with PBS twice, fixed with $4 \%$ paraformaldehyde for $10 \mathrm{~min}$ at room temperature and lysed in hot lysis buffer (HLB; $1 \%$ SDS, $1 \mathrm{mM}$ EDTA and $2 \mathrm{mM} \mathrm{Na}_{3} \mathrm{VO}_{4}$ ) in boiled water for $5 \mathrm{~min}$. Subsequent to ultrasonic treatment, lysates were clarified by centrifuge and diluted with an equivalent volume of immunomix special solution (IMS; $2 \%$ Triton $X$ $100,0.5 \%$ sodium deoxycholate, $1 \%$ BSA, $1 \mathrm{mM}$ EDTA, $2 \mathrm{mM} \mathrm{Na}_{3} \mathrm{VO}_{4}$ and $0.5 \%$ protease inhibitor cocktail (PIC; Roche)), and subjected to immunoprecipitation. For the preparation of endogenous protein complexes including the PML complex, cells were lysed in high-salt immunoprecipitation buffer (HSB; $400 \mathrm{mM} \mathrm{NaCl}, 50 \mathrm{mM}$ HEPES-Na, $\mathrm{pH}$ 7.0, $1 \mathrm{mM}$ EDTA, $0.1 \% \mathrm{NP}-40$ and $0.5 \% \mathrm{PIC}$ ), and the lysate was clarified by centrifuge. Protein concentration was quantitated by bicinchoninic acid assay $^{36}$ using a BCA-1 protein assay kit (Sigma-Aldrich, St. Louis, MO, USA).

For immunoprecipitation, primary antibodies were fixed on either protein A- or protein G-sepharose beads. ${ }^{33}$ Briefly, $10 \mu \mathrm{g}$ of primary antibody bound to either beads was crosslinked in $100 \mathrm{mM}$ sodium borate buffer containing $5 \mathrm{mM}$ dimethyl pimelimidate at $\mathrm{pH} 8.5$ for $30 \mathrm{~min}$, and blocked by $200 \mathrm{mM}$ of ethanolamine for $2 \mathrm{~h}$. The fixed antibody beads were suspended in PBS at $10 \%$ and $2 \mu$ of the suspension was applied to cell lysate corresponding to $1 \mathrm{mg}$ of protein. Immune complex was formed at $4^{\circ} \mathrm{C}$ for $3 \mathrm{~h}$ with constant agitation, and was washed four times with HSB. The antigen was released in $30 \mu$ of elution buffer (IPE; $50 \mathrm{mM}$ Tris$\mathrm{HCl}, \mathrm{pH} 6.8,1 \% \mathrm{SDS}$ and $1 \% \mathrm{DOC}-\mathrm{Na}$ ) at $42^{\circ} \mathrm{C}$ for $30 \mathrm{~min}$.

For immunoblotting, antigen released from the $\mathrm{IP}$, or $20 \mu \mathrm{g}$ of WCL, was separated by conventional SDS-PAGE, and transferred to a nylon membrane. To detect the antigen, blots were blocked in TBS-T $(25 \mathrm{mM}$ Tris- $\mathrm{HCl}, \mathrm{pH} 8.0,137 \mathrm{mM} \mathrm{NaCl}, 2.7 \mathrm{mM} \mathrm{KCl}$ and $0.1 \%$ Tween 20) containing $5 \%$ skim milk (Difco) at room temperature for $1 \mathrm{~h}$, and incubated with the primary antibody for $2 \mathrm{~h}$. The blots were then incubated with secondary antibody labeled with either alkaline phosphatase (AP) or horseradish peroxidase (HRP) at room temperature for $1 \mathrm{~h}$. Immune complexes were detected with CDP-Star reagent or Lumi-LightPLUS Western blotting substrate (Roche) according to the manufacturer's directions. The following antibodies were used: mouse anti-E2F-1 (Phamingen), rabbit anti-E2F-2 (C-20) (Santa Cruz, Santa Cruz, CA, USA), rabbit anti-E2F-4 (C-20) (Santa Cruz), mouse anti-HA (12CA5) (Roche), mouse anti-FLAG M5 (Sigma), mouse anti-PML (PG-M3) (Santa Cruz), rabbit anti-PML (H-238) (Santa Cruz), rabbit anti-E2FBP1, ${ }^{1}$ rabbit anti-Sp100 (Chemicon, Temecula, CA, USA), rabbit anti-p53 (FL393) (Santa Cruz), mouse anti-p53 (DO-1) (Santa Cruz), rabbit anti-SUMO-1 (FL101) (Santa Cruz), rabbit anti-p16 (C-20) (Santa Cruz), rabbit anti-p21 (Santa Cruz) and rabbit anti-PAl-1 (H-135) (Santa Cruz). 


\section{Immunofluorescent microscopy}

Cells grown on cover slips were transfected with expression plasmids and fixed for 15 min with fresh culture medium containing $3.7 \%$ formaldehyde at $37^{\circ} \mathrm{C}$. The fixed cells were treated with PBS containing $0.3 \%$ Triton X100 for $5 \mathrm{~min}$, washed twice with PBS and blocked for $2 \mathrm{~h}$ by PBS containing $1 \%$ FCS. The cells were then stained with primary antibodies at room temperature for $3 \mathrm{~h}$, and visualized with fluorochrome-labeled secondary antibodies plus $0.1 \mu \mathrm{g} / \mathrm{ml}$ of Hoechst 33342 . Fluorescent microscopy was performed using a Zeiss laser-scanning confocal microscope system with LSM510 software version 2.3 (Carl Zeiss, Germany). The following antibodies were used: rabbit anti-E2FBP1, ${ }^{1}$ FITC-conjugated goat anti-mouse IgG-Fc (Cappel), Cy3-conjugated donkey anti-mouse $\lg G \mathrm{~F}\left(\mathrm{ab}^{\prime}\right)_{2}$ and Cy3-conjugated donkey anti-rabbit $\operatorname{lgG} F\left(a b^{\prime}\right)_{2}$ (Jackson ImmunoResearch, West Grove, PA, USA) diluted at $1: 500$, mouse anti-PML (PG-M3) (Santa Cruz) diluted at 1:100.

\section{In vitro dissociation assay}

GST-Ulp1 and rE2FBP1 (i.e. (His) ${ }_{6}$-E2FBP1) expressed in E. coli were purified on a glutathione-sepharose column or $\mathrm{Ni}$ chelating column. rE2FBP1 was further purified with a heparin-sepharose column based on the feature of a DNA-binding factor to minimize the contamination. Both proteins were stored in desalting buffer $(50 \mathrm{mM}$ Tris- $\mathrm{HCl}, \mathrm{pH} 7.4,5 \mathrm{mM}$ $\mathrm{MgCl}_{2}$ and $10 \% \mathrm{glycerol}$ ) at $0.5 \mu \mathrm{g} / \mu \mathrm{l}$. The PML complex was prepared as stated above. The reaction was performed in $50 \mu \mathrm{l}$ of reaction buffer (50 mM Tris- $\mathrm{HCl}, \mathrm{pH} 7.4,5 \mathrm{mM} \mathrm{MgCl}_{2}$ and $2 \mathrm{mM}$ DTT). PML complex corresponding to $650 \mu \mathrm{g}$ of cell lysate was incubated with or without rE2FBP1 or GST-Ulp1 at $32^{\circ} \mathrm{C}$ for $1 \mathrm{~h}$, and boiled for $5 \mathrm{~min}$ with an equivalent volume of $2 \times$ Laemmli's sample buffer (4\% SDS, $120 \mathrm{mM}$ Tris- $\mathrm{HCl}, \mathrm{pH} 6.8,10 \%$ glycerol, and $250 \mathrm{mM}$ of freshly prepared DTT). One-third of each sample was subjected to SDS-PAGE and subsequently to immunoblotting.

\section{siRNA-mediated downregulation}

The target sequence for siRNA-E was as follows: (sense) $5^{\prime}$ GCAGCUCUACGAACUCGACTT-3', (antisense) 5'-GUCGAGUUCGUAGAGCUGCTT-3'. siRNA-N was designed as stated in the text: (sense) $5^{\prime}$ GCUUGAGCCGCGGAAUUCCTT-3', (antisense) 5'-GGAAUUCCGCGGCUCAAGCTT- $3^{\prime}$. siRNA-P was a mixture of two pairs of siRNAs against $\mathrm{PML}$ and the target sequence chosen according to $\mathrm{Xu}$ et $\mathrm{al}^{32}$ was as follows: (sense-1) 5'-GCACGAAGACAGACCUCUGTT-3', (antisense-1) 5'-CAGAGGUCUGUCUUCGUGCTT-3' and (sense-2) 5'-CGACAGCCCAGAAGAGGAA-3', (antisense-2) 5'-CCUCUUCUGGGCUGUCGTT-3'. siRNAs were synthesized by Japan Bioservice Co. (Saitama) and annealed at a concentration of $40 \mu \mathrm{M}$ in annealing buffer $(100 \mathrm{mM}$ potassium acetate, $30 \mathrm{mM}$ HEPES-KOH, $\mathrm{pH} 7.4$, and $2 \mathrm{mM}$ magnesium acetate). Transfection was performed with oligofectamine (Life Technologies), and cells were treated with $0.2 \mu \mathrm{M}$ of respective ds-siRNA.

To perform growth analysis, TIG-3 cells at 36 PD were plated at $1 \times 10^{6}$ per 35-mm dish in triplicate and treated with either siRNA or mock reagent within $12 \mathrm{~h}$ after passage. Cells were divided into two, one aliquot was used for cell counting and the other treated similarly with either siRNA or mock reagent.

\section{SA- $\beta$-Gal assay}

The SA- $\beta$-Gal assay was performed as described. ${ }^{31}$ Briefly, 2 days after the last treatment with SiRNA, TIG-3 cells were fixed with $3 \%$ formaldehyde for $5 \mathrm{~min}$, washed, and incubated at $37^{\circ} \mathrm{C}$ with staining buffer $(1 \mathrm{mg} / \mathrm{ml}$ 5-bromo-4-chloro-3-indolyl- $\beta$-D-galactopiranoside, $5 \mathrm{mM}$ potassium ferrocyanide, $5 \mathrm{mM}$ potassium ferricyanide, $150 \mathrm{mM} \mathrm{NaCl}$, $2 \mathrm{mM} \mathrm{MgCl}_{2}$ and $40 \mathrm{mM}$ citric acid/sodium phosphate, $\mathrm{pH} \mathrm{6.0)} \mathrm{for} 12 \mathrm{~h}$.

\section{Acknowledgements}

We thank $Y$ Takahashi and $Y$ Kikuchi for discussions, the HA-tag DNA fragment and the expression plasmid for GST-UIp1. This work was partly supported by a Grant-in Aid for Scientific Research from the Japan Society for the Promotion of Science.

\section{References}

1. Suzuki $M$, Okuyama $S$, Okamoto $S$, Shirasuna $K$, Nakajima $T$, Hachiya $T$, Nojima H, Sekiya S and Oda K (1998) A novel E2F binding protein with Myctype HLH motif stimulates E2F-dependent transcription by forming a heterodimer. Oncogene 17: 853-865

2. Kortschak RD, Reimann H, Zimmer M, Eyre HJ, Saint R and Jenne DE (1998) The human dead ringer/bright homolog, DRIL1: cDNA cloning, gene structure, and mapping to D19S886, a marker on 19p13.3 that is strictly linked to the Peutz-Jeghers syndrome. Genomics 51: 288-292

3. Kortschak RD, Tucker PW and Saint R (2000) ARID proteins come in from the desert. Trends Biochem. Sci. 25: 294-299

4. Gong L, Kamitani T, Fujise K, Caskey LS and Yeh ET (1997) Preferential interaction of sentrin with a ubiquitin-conjugating enzyme, Ubc9. J. Biol. Chem. 272: 28198-28201

5. Zong RT, Das C and Tucker PW (2000) Regulation of matrix attachment region-dependent, lymphocyte-restricted transcription through differential localization within promyelocytic leukemia nuclear bodies. EMBO J. 19: 4123-4133

6. Webb CF (2001) The transcription factor, Bright, and immunoglobulin heavy chain expression. Immunol. Res. 24: 149-161

7. Herrscher RF, Kaplan MH, Lelsz DL, Das C, Scheuermann R and Tucker PW (1995) The immunoglobulin heavy-chain matrix-associating regions are bound by Bright: a B cell-specific trans-activator that describes a new DNA-binding protein family. Genes Dev. 9: 3067-3082

8. Borden KL (2002) Pondering the promyelocytic leukemia protein (PML) puzzle: possible functions for PML nuclear bodies. Mol. Cell Biol. 22: 5259-5269

9. Maul GG, Negorev D, Bell P and Ishov AM (2000) Review: properties and assembly mechanisms of ND10, PML bodies, or PODs. J. Struct. Biol. 129: 278-287

10. Negorev D and Maul GG (2001) Cellular proteins localized at and interacting within ND10/PML nuclear bodies/PODs suggest functions of a nuclear depot. Oncogene 20: 7234-7242

11. Seeler JS and Dejean A (1999) The PML nuclear bodies: actors or extras? Curr. Opin. Genet. Dev. 9: 362-367

12. Eskiw CH and Bazett-Jones DP (2002) The promyelocytic leukemia nuclear body: sites of activity? Biochem. Cell Biol. 80: 301-310

13. Pearson M and Pelicci PG (2001) PML interaction with $p 53$ and its role in apoptosis and replicative senescence. Oncogene 20: 7250-7256

14. Salomoni P and Pandolfi PP (2002) The role of PML in tumor suppression. Cell 108: $165-170$

15. Everett RD (2001) DNA viruses and viral proteins that interact with PML nuclear bodies. Oncogene 20: 7266-7273

16. Benoit G, Roussel M, Pendino F, Segal-Bendirdjian E and Lanotte M (2001) Orchestration of multiple arrays of signal cross-talk and combinatorial interactions for maturation and cell death: another vision of $t(15 ; 17)$ preleukemic blast and APL-cell maturation. Oncogene 20: 7161-7177

17. Pearson M, Carbone R, Sebastiani C, Cioce M, Fagioli M, Saito S, Higashimoto Y, Appella E, Minucci S, Pandolfi PP and Pelicci PG (2000) PML regulates p53 acetylation and premature senescence induced by oncogenic Ras. Nature 406 : $207-210$ 
18. Ferbeyre G, de Stanchina E, Querido E, Baptiste N, Prives C and Lowe SW (2000) PML is induced by oncogenic ras and promotes premature senescence. Genes Dev. 14: 2015-2027

19. James P, Halladay J and Craig EA (1996) Genomic libraries and a host strain designed for highly efficient two-hybrid selection in yeast. Genetics 144: 1425-1436

20. Fogal V, Gostissa M, Sandy P, Zacchi P, Sternsdorf T, Jensen K, Pandolfi PP Will H, Schneider C and Del Sal G (2000) Regulation of p53 activity in nuclear bodies by a specific PML isoform. EMBO J. 19: 6185-6195

21. Everett RD, Lomonte $P$, Sternsdorf T, van Driel R and Orr A (1999) Cell cycle regulation of PML modification and ND10 composition. J. Cell Sci. 112 (Part 24): $4581-4588$

22. Zhong S, Müller S, Ronchetti S, Freemont PS, Dejean A and Pandolfi PP (2000) Role of SUMO-1-modified PML in nuclear body formation. Blood 95: 2748-2752

23. Ishov AM, Sotnikov AG, Negorev D, Vladimirova OV, Neff N, Kamitani T, Yeh ET, Strauss 3rd JF and Maul GG (1999) PML is critical for ND10 formation and recruits the PML-interacting protein daxx to this nuclear structure when modified by SUMO-1. J. Cell Biol. 147: 221-234

24. Best JL, Ganiatsas S, Agarwal S, Changou A, Salomoni P, Shirihai O, Meluh PB, Pandolfi PP and Zon LI (2002) SUMO-1 protease-1 regulates gene transcription through PML. Mol. Cell 10: 843-855

25. Gostissa M, Hengstermann A, Fogal V, Sandy P, Schwarz SE, Scheffner M and Del Sal G (1999) Activation of p53 by conjugation to the ubiquitin-like protein SUMO-1. EMBO J. 18: 6462-6471

26. Rodriguez MS, Desterro JM, Lain S, Midgley CA, Lane DP and Hay RT (1999) SUMO-1 modification activates the transcriptional response of p53. EMBO J. 18: 6455-6461

27. Li S-J and Hochstrasser M (1999) A new protease required for cell-cycle progression in yeast. Nature 398: 246-251
28. Hannon GJ (2002) RNA interference. Nature 418: 244-251

29. Ohtani N, Zebedee Z, Huot TJ, Stinson JA, Sugimoto M, Ohashi $Y$, Sharrocks AD, Peters G and Hara E (2001) Opposing effects of Ets and Id proteins on p16INK4a expression during cellular senescence. Nature 409: 1067-1070

30. Serrano M, Lin AW, McCurrach ME, Beach D and Lowe SW (1997) Oncogenic ras provokes premature cell senescence associated with accumulation of p53 and p16INK4a. Cell 88: 593-602

31. Dimri GP, Lee X, Basile G, Acosta M, Scott G, Roskelley C, Medrano EE, Linskens M, Rubelij I, Pereira-Smith $O$, Peacocke $M$ and Campisi J (1995) A biomarker that identifies senescent human cells in culture and in aging skin in vivo. Proc. Natl. Acad. Sci. USA 92: 9363-9367

32. Xu ZX, Timanova-Atanasova A, Zhao RX and Chang KS (2003) PML colocalizes with and stabilizes the DNA damage response protein TopBP1. Mol. Cell Biol. 23: 4247-4256

33. Harlow $E$ and Lane D (1999) Immunoaffinity purification. In Using Antibodies; A Laboratory Manual, 1st edn. (New York: Cold Spring Harbor Laboratory) pp. 311-343

34. Kaplan MH, Zong RT, Herrscher RF, Scheuermann RH and Tucker PW (2001) Transcriptional activation by a matrix associating region-binding protein. Contextual requirements for the function of bright. J. Biol. Chem. 276: 21325-21330

35. Peeper DS, Shvarts A, Brummelkamp T, Douma S, Koh EY, Daley GQ and Bernards R (2002) A functional screen identifies hDRIL1 as an oncogene that rescues RAS-induced senescence. Nat. Cell Biol. 4: 148-153

36. Smith P, Krohn R, Hermanson G, Mallia A, Gartner F, Provenzano M, Fujimoto E, Goeke N, Olson B and Klenk D (1985) Measurement of protein using bicinchoninic acid. Anal. Biochem. 150: 76-85 